\title{
Higher Order Runge-Kutta Method for Solving Fuzzy Differential Equations
}

\author{
Fuziyah Ishak* and Nor Salihah Zakaria \\ Faculty of Computer and Mathematical Sciences, Universiti Teknologi MARA, 40450 Shah Alam, \\ Selangor, Malaysia
}

\begin{abstract}
Real world problems are often described using mathematical models. The construction of these models often give rise to fuzzy differential equations where imprecise and vagueness are common in real world. Finding real world solutions require finding solutions to various type of fuzzy differential equations, which have gained popularity in modelling real life phenomena. Most of fuzzy differential equations that describe real world problems cannot be solved analytically. Thus, researchers focus on finding numerical methods that can be made as accurately as possible. Numerical methods for solving ordinary differential equations can be adapted to solve fuzzy differential equations. In this study, we aim to implement Runge-Kutta method of order six to solve wide ranges of fuzzy differential equations. The efficiency and accuracy of the method will be tested using various stepsizes. Numerical results will be further tested against existing methods in literatures. The implementation of Runge-Kutta method of higher order proves to be more accurate and able to solve various types of fuzzy differential equations.
\end{abstract}

Keywords: fuzzy and vagueness, mathematical models, fuzzy differential equations, numerical solutions, Runge-Kutta methods, accuracy

\section{INTRODUCTION}

Mathematical models are often used to describe many real-world problems. The construction of these models give rise to fuzzy differential equations (FDEs) where imprecise and vagueness are common in real world. The study of FDEs is one of the new branches of mathematics, which has expanded very quickly in recent years. Its theories and applications have been discussed throughout time by many researchers, which among others are Zadeh (1965), Bede et al. (2007), Zimmerman (2010), Jayakumar et al. (2012) and Sulaiman et al. (2018).

Most FDEs cannot be solved analytically, thus give rise to numerical methods that can be made as accurately as possible. Numerical methods to solve FDEs are among those developed by Abbasbandy and Viranloo (2002), Kanagarajan and Sambath (2010), Ahmad et al. (2013),
Jayakumar et al. (2014) and Ismail et al. (2017). Most of the numerical methods to solve FDEs are adapted from numerical methods for solving ordinary differential equations (ODEs), see for example Khairul Anuar (2011) for numerical methods in solving ODEs. Among the popular methods are Runge-Kutta (RK) type of methods. These methods, which are an important family of implicit and explicit iterative methods are often studied and implemented for the approximation of numerical solutions for various types of differential equations. According to Baier et al. (2012), RK methods or higher order can be made to calculate a good approximation of the jumps. As for numerical methods for solving FDEs, various researchers implemented RK methods of lower order and concentrated on RK methods of order four, as in Palligkinis et al. (2009). Moreover, Kanagarajan and Sambath (2010) and Jayakumar et al. (2012) have both

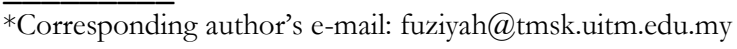


worked on the implementation of RK methods of order three and order five respectively for solving initial values FDEs. These methods provide accurate solution and comparably easy to be implemented. Numerical methods of higher order will give better accuracy and have been studied widely to solve many types of differential equations. One-step method such as RK type of methods can be implemented efficiently with minimal additional computational costs as the previous RK methods of lower order. Through extensive literature review, RK methods have been adapted to solve FDEs but to our knowledge of up to date, no RK method of order six (RK6) has been applied to solve FDEs, please refer to Jayakumar et al. (2012)

The motivation of this research is to implement RK6 for solving FDEs. Higher order method will gain better accuracy than previous methods of lower order and since the method belongs to the family of one-step method, computational complexity and efficiency will not be of major cost issues, i.e. in terms of computational cost. The numerical results of RK6 will be compared in terms of accuracy with both Euler method and RK method of order five ( $\left.\mathrm{RK}_{5}\right)$ in Jayakumar et al. (2012).

The organization of this paper is as follows. In the next section, we state some preliminaries that are fundamentals to FDEs and describe the method of RK6 and its implementation in solving initial value FDEs. The following section is followed by some numerical results for RK6 as well as Euler method and RK5 with discussions that include error analysis. The last section highlights the conclusion.

\section{MATERIALS AND METHODS}

\section{Preliminaries}

In this section, we introduce some definitions and basic preliminaries regarding FDEs.

Definition 1. (Bede (2013)) A fuzzy set A (fuzzy subset of $X)$ is defined as a mapping $A: X \rightarrow[0,1]$, where $A(x)$ is the membership degree of $x$ to the fuzzy subset $A$. We denote by $F(X)$ the collection of all fuzzy subset of $X$.
Definition 2. Consider a fuzzy subset of the real line $u: R \rightarrow[0,1]$. Then $u$ is a fuzzy number if it satisfies the following properties:

i) $u$ is normal, i.e. there exists $x_{\mathrm{o}} \in R$ with $u\left(x_{\mathrm{o}}\right)=1$

ii) $u$ is fuzzy convex, i.e. $u(t x+(1-t) y) \geq \min \{u(x), u(y)\}$ $t \in[0,1], x, y \in R$;

iii) $u$ is upper semi continuous on $R$, i.e. for every $\varepsilon>0$, there exists $\delta>0$ such that $u(x)-u\left(x_{0}\right)<\varepsilon,\left|x-x_{0}\right|<\delta$.

iv) $u$ is compactly supported, i.e. $\operatorname{cl}\{x \in R, u(x)>0\}$ is compact, where $\operatorname{cl}(A)$ denotes the closure of the set $A$.

We denote by $R_{F}$ the space of fuzzy numbers.

Definition 3. (Bede (2013) and Jayakumar et al. (2012)) For $0<r \leq 1$, we denote $[u]_{r}=\{x \in R: u(x) \geq r\}$ and $[u]_{0}=c l\{x \in R: u(x)>0\}$. Then $[u]_{r}$ is called the $r$ level set of the fuzzy number $u$. Moreover $[u]_{r}$ is a closed interval $[u]_{r}=\left[u_{1}(r), u_{2}(r)\right]$, for any $r \in[0,1]$.

Definition 4. A triangular fuzzy number $u$ is represented by a triplet $(a, b, c) \in R^{3}, a \leq b \leq c$ where

$$
u(t)\left\{\begin{array}{l}
0, \text { if } t<a \\
\frac{t-a}{b-a}, \text { if } a \leq t \leq b \\
1, \text { if } t=b \\
\frac{c-t}{c-b}, \text { if } b \leq t \leq c \\
o, \text { if } c<t
\end{array}\right.
$$

Let $I$ be a real interval. A mapping $y: I \rightarrow R_{F}$ is called a fuzzy process and its $r$-level set is denoted by $[y(t)]_{r}=\left[y_{1}(t ; r), y_{2}(t ; r)\right], t \in I, r \in[0,1] . \quad$ The Seikkala derivative (Seikkala, 1987) $y^{\prime}(t)$ of the fuzzy process $y(t)$ is given by $\left[y^{\prime}(t)\right]_{r}=\left[y_{1}^{\prime}(t ; r), y_{2}^{\prime}(t ; r)\right]$, $t \in I, r \in[0,1]$, provided this equation defines a fuzzy number.

Lemma 1. (Jayakumar et al. (2012)) For $v, w \in R_{F}$ 
and $s$ a scalar and $r \in[0,1]$, then

i) $[v+w]_{r}=\left[v_{1}(r)+w_{1}(r), v_{2}(r)+w_{2}(r)\right]$,

ii) $[v-w]_{r}=\left[v_{1}(r)-w_{1}(r), v_{2}(r)-w_{2}(r)\right]$,

$$
[v \cdot w]_{r}=\left[\operatorname { m i n } \left\{v_{1}(r) \cdot w_{1}(r), v_{1}(r) \cdot w_{2}(r),\right.\right.
$$

iii) $\left.v_{2}(r) . w_{1}(r), v_{2}(r) . w_{2}(r)\right\}$

$$
\begin{array}{r}
\max \left\{v_{1}(r) \cdot w_{1}(r), v_{1}(r) \cdot w_{2}(r),\right. \\
\left.\left.v_{2}(r) \cdot w_{1}(r), v_{2}(r) \cdot w_{2}(r)\right\}\right],
\end{array}
$$

iv) $[s v]_{r}=s[v]_{r}$.

\section{Method Implementation}

We consider the fuzzy initial value problem as follows:

$$
\left\{\begin{array}{l}
y^{\prime}(t)=f(t, y(t), t \in[0, T] \\
y(0)=y_{0}
\end{array}\right.
$$

where $f$ is a continuous mapping from $R^{+} \times R$ into $R$ and $y_{\mathrm{o}} \in R_{F}$ with $r$-level sets $\left[y_{\mathrm{o}}\right]_{r}=\left[y_{1}(\mathrm{o} ; r), y_{2}(\mathrm{o} ; r)\right]$, $r \in[0,1]$.Zadeh's Extension Principle, Zadeh (1975) leads to the following definition of $f(t, y)$ when $y=y(t)$ is a fuzzy number

$$
f(t, y)(s)=\sup \{y(\tau) \mid s=f(t, \tau)\}, s \in R .
$$

It follows that

$$
[f(t, y)]_{r}=\left[f_{1}(t, y ; r), f_{2}(t, y ; r)\right], r \in[0,1],
$$

where

$$
f_{1}(t, y ; r)=\min \left\{f(t, u) \mid u \in\left[y_{1}(r), y_{2}(r)\right]\right\},
$$

and

$$
f_{2}(t, y ; r)=\max \left\{f(t, u) \mid u \in\left[y_{1}(r), y_{2}(r)\right]\right\} .
$$

Furthermore, $f$ is bounded and satisfies Lipschitz condition that guarantees the existence of a unique solution. We consider uniform grid with stepsize $h=\frac{T}{N}$ such that $t_{n}=n h, n=0,1, \ldots, N . \quad$ The exact and approximate solutions at $t_{n}$ are denoted by $\left[Y\left(t_{n}\right)\right]_{r}=\left[Y_{1}\left(t_{n} ; r\right), Y_{2}\left(t_{n} ; r\right)\right]$ and $\left[y\left(t_{n}\right)\right]_{r}=\left[y_{1}\left(t_{n} ; r\right), y_{2}\left(t_{n} ; r\right)\right]$ respectively. Thus, RK6 for solving FDEs are given by the following:

$y_{1}\left(t_{n+1}, r\right)-y_{1}\left(t_{n}, r\right)=\sum_{i=1}^{7} w_{i} k_{i, 1}$ and $y_{2}\left(t_{n+1}, r\right)-y_{2}\left(t_{n}, r\right)=\sum_{i=1}^{7} w_{i} k_{i, 2} \quad$ where $w_{i}, i=1,2, \ldots, 7$ are constants. The $k_{i}$ are given by $\left[k_{i}\left(t_{n}, y\left(t_{n} ; r\right)\right]_{r}=\left[k_{i, 1}\left(t_{n}, y\left(t_{n} ; r\right), k_{i, 2}\left(t_{n}, y\left(t_{n} ; r\right)\right)\right]\right.\right.$ and

$$
\begin{gathered}
k_{1,1}\left(t_{n}, y\left(t_{n} ; r\right)\right)=h f\left(t_{n}, y_{1}\left(t_{n} ; r\right)\right) \\
k_{1,2}\left(t_{n}, y\left(t_{n} ; r\right)\right)=h f\left(t_{n}, y_{2}\left(t_{n} ; r\right)\right) \\
k_{2,1}\left(t_{n}, y\left(t_{n} ; r\right)\right)=h f\left(t_{n}+h, y_{1}\left(t_{n} ; r\right)+k_{1,1}\right) \\
k_{2,2}\left(t_{n}, y\left(t_{n} ; r\right)\right)=h f\left(t_{n}+h, y_{2}\left(t_{n} ; r\right)+k_{1,2}\right) \\
k_{3,1}\left(t_{n}, y\left(t_{n} ; r\right)\right)=h f\left(t_{n}+\frac{h}{2}, y_{1}\left(t_{n} ; r\right)+\frac{3 k_{1,1}+k_{2,1}}{8}\right)
\end{gathered}
$$

$k_{3,2}\left(t_{n}, y\left(t_{n} ; r\right)\right)=h f\left(t_{n}+\frac{h}{2}, y_{2}\left(t_{n} ; r\right)+\frac{3 k_{1,2}+k_{2,2}}{8}\right)$

$k_{4,1}\left(t_{n}, y\left(t_{n} ; r\right)\right)=h f\left(t_{n}+\frac{2 h}{3}, y_{1}\left(t_{n} ; r\right)+\frac{8 k_{1,1}+2 k_{2,1}+8 k_{3,1}}{27}\right)$

$k_{4,2}\left(t_{n}, y\left(t_{n} ; r\right)\right)=h f\left(t_{n}+\frac{2 h}{3}, y_{2}\left(t_{n} ; r\right)+\frac{8 k_{1,2}+2 k_{2,2}+8 k_{3,2}}{27}\right)$ and $k_{5,1}, k_{5,2}, \ldots, k_{7,1}, k_{7,2}$ are as given in Butcher (1963), Luther (1967) and Zakaria (2016). Thus, the formulae for the approximate values at the grid are given by

$$
\begin{aligned}
& y_{1}\left(t_{n+1} ; r\right)=y_{1}\left(t_{n} ; r\right) \\
& \quad+\frac{1}{180}\left(9 k_{1,1}+64 k_{3,1}+49 k_{5,1}+49 k_{6,1}+9 k_{7,1}\right)
\end{aligned}
$$

and

$$
\begin{aligned}
& y_{2}\left(t_{n+1} ; r\right)=y_{2}\left(t_{n} ; r\right) \\
& \quad+\frac{1}{180}\left(9 k_{1,2}+64 k_{3,2}+49 k_{5,2}+49 k_{6,2}+9 k_{7,2}\right)
\end{aligned}
$$

\section{RESULTS AND DISCUSSIONS}

For illustrative purposes, we show numerical results for two test problems. The test problems are taken from Kanagarajan and Sambath (2010) and Jayakumar et al. (2012)). Numerical experiments are carried out to compute approximate values at grid points using various values for stepsize where the errors against exact solutions are computed and compared. Numerical results of RK6 as explained in the previous section are also compared with RK5 and Euler methods. 
Test Problem 1:

$\left\{\begin{array}{l}y^{\prime}(t)=y(t), t \in[0,1] \\ y(0)=(0.75+0.25 r, 1.125-0.125 r), 0<r \leq 1\end{array}\right.$

The exact solution is given by $Y_{1}(t ; r)=y_{1}(0 ; r) e^{t}$ and $Y_{2}(t ; r)=y_{2}(0 ; r) e^{t}$, at which at $t=1$, $Y_{1}(1 ; r)=y_{1}(0.75+0.25 r) e$ and $Y_{2}(1 ; r)=y_{1}(1.125-0.125 r) e, 0<r \leq 1$.

Test Problem 2: $\left\{\begin{array}{l}y^{\prime}(t)=t y(t), t \in[0,1], \\ y(0)=(\sqrt{e}-0.5(1-r), \sqrt{e}+0.5(1-r), 0<r \leq 1\end{array}\right.$
The exact solution is given by

$Y_{1}(t ; r)=(\sqrt{e}-0.5(1-r)) e^{t^{2} / 2}$ and

$Y_{2}(t ; r)=(\sqrt{e}+0.5(1-r)) e^{t^{2} / 2}$ at which at $t=1$,

$Y_{1}(1 ; r)=(\sqrt{e}-0.5(1-r)) e^{0.5}$ and

$Y_{2}(1 ; r)=(\sqrt{e}+0.5(1-r)) e^{0.5}$

Numerical results of RK6 at $t=1$ in terms of absolute errors for $h=0.1, h=0.01$ and $h=0.001$, and $r=0.1,0.2, \ldots, 1.0$ for Test Problem1 and Test Problem 2 are given in Table 1 and Table 2 respectively. Referring to the tables, the value $1.425 e-09$ means $1.425 \times 10^{-9}$

Table 1: Absolute Errors of Test Problem 1 using RK6 with Different Values of $h$

\begin{tabular}{|c|c|c|c|c|c|c|}
\hline \multirow{2}{*}{$r$} & \multicolumn{5}{|c|}{ Absolute Errors for RK6 } \\
\cline { 2 - 8 } & \multicolumn{2}{|c|}{$h=0.1$} & \multicolumn{2}{c|}{$h=0.01$} & \multicolumn{2}{c|}{$h=0.001$} \\
\cline { 2 - 8 } & $\left|Y_{1}-y_{1}\right|$ & $\left|Y_{2}-y_{2}\right|$ & $\left|Y_{1}-y_{1}\right|$ & $\left|Y_{2}-y_{2}\right|$ & $\left|Y_{1}-y_{1}\right|$ & $\left|Y_{2}-y_{2}\right|$ \\
\hline 0.1 & $1.425 \mathrm{e}-09$ & $2.046 \mathrm{e}-09$ & $1.632 \mathrm{e}-12$ & $2.347 \mathrm{e}-12$ & $2.176 \mathrm{e}-14$ & $1.910 \mathrm{e}-14$ \\
\hline 0.2 & $1.471 \mathrm{e}-09$ & $2.023 \mathrm{e}-09$ & $1.686 \mathrm{e}-12$ & $2.321 \mathrm{e}-12$ & $2.354 \mathrm{e}-14$ & $2.132 \mathrm{e}-14$ \\
\hline 0.3 & $1.517 \mathrm{e}-09$ & $2.000 \mathrm{e}-09$ & $1.739 \mathrm{e}-12$ & $2.291 \mathrm{e}-12$ & $2.531 \mathrm{e}-14$ & $3.419 \mathrm{e}-14$ \\
\hline 0.4 & $1.563 \mathrm{e}-09$ & $1.977 \mathrm{e}-09$ & $1.792 \mathrm{e}-12$ & $2.266 \mathrm{e}-12$ & $1.821 \mathrm{e}-14$ & $2.620 \mathrm{e}-14$ \\
\hline 0.5 & $1.609 \mathrm{e}-09$ & $1.954 \mathrm{e}-09$ & $1.846 \mathrm{e}-12$ & $2.242 \mathrm{e}-12$ & $2.354 \mathrm{e}-14$ & $2.265 \mathrm{e}-14$ \\
\hline 0.6 & $1.655 \mathrm{e}-09$ & $1.931 \mathrm{e}-09$ & $1.898 \mathrm{e}-12$ & $2.215 \mathrm{e}-12$ & $2.176 \mathrm{e}-14$ & $2.087 \mathrm{e}-14$ \\
\hline 0.7 & $1.701 \mathrm{e}-09$ & $1.908 \mathrm{e}-09$ & $1.950 \mathrm{e}-12$ & $2.186 \mathrm{e}-12$ & $1.865 \mathrm{e}-14$ & $2.531 \mathrm{e}-14$ \\
\hline 0.8 & $1.747 \mathrm{e}-09$ & $1.885 \mathrm{e}-09$ & $2.000 \mathrm{e}-12$ & $2.164 \mathrm{e}-12$ & $2.531 \mathrm{e}-14$ & $2.798 \mathrm{e}-14$ \\
\hline 0.9 & $1.793 \mathrm{e}-09$ & $1.862 \mathrm{e}-09$ & $2.057 \mathrm{e}-12$ & $2.134 \mathrm{e}-12$ & $1.865 \mathrm{e}-14$ & $2.087 \mathrm{e}-14$ \\
\hline 1.0 & $1.839 \mathrm{e}-09$ & $1.839 \mathrm{e}-09$ & $2.109 \mathrm{e}-12$ & $2.109 \mathrm{e}-12$ & $2.087 \mathrm{e}-14$ & $2.087 \mathrm{e}-14$ \\
\hline
\end{tabular}

Table 2: Absolute Errors of Test Problem 2 using RK6 with Different Values of $h$

\begin{tabular}{|c|c|c|c|c|c|c|}
\hline \multicolumn{7}{|c|}{ Absolute Errors for RK6 } \\
\hline \multirow[t]{2}{*}{$r$} & \multicolumn{2}{|c|}{$h=0.1$} & \multicolumn{2}{|c|}{$h=0.01$} & \multicolumn{2}{|c|}{$h=0.001$} \\
\hline & $\left|Y_{1}-y_{1}\right|$ & $\left|Y_{2}-y_{2}\right|$ & $\left|Y_{1}-y_{1}\right|$ & $\left|Y_{2}-y_{2}\right|$ & $\left|Y_{1}-y_{1}\right|$ & $\left|Y_{2}-y_{2}\right|$ \\
\hline 0.1 & $2.834 \mathrm{e}-08$ & $4.962 \mathrm{e}-08$ & $9.370 \mathrm{e}-14$ & $1.625 \mathrm{e}-13$ & $1.288 \mathrm{e}-14$ & $1.155 \mathrm{e}-14$ \\
\hline 0.2 & $2.953 \mathrm{e}-08$ & $4.844 \mathrm{e}-08$ & $9.548 \mathrm{e}-14$ & $1.603 \mathrm{e}-13$ & $1.776 \mathrm{e}-15$ & $1.332 \mathrm{e}-14$ \\
\hline 0.3 & $3.071 \mathrm{e}-08$ & $4.726 \mathrm{e}-08$ & $1.008 \mathrm{e}-13$ & $1.537 \mathrm{e}-13$ & $3 \cdot 553 \mathrm{e}-15$ & $1.110 \mathrm{e}-14$ \\
\hline 0.4 & $3.189 \mathrm{e}-08$ & $4.608 \mathrm{e}-08$ & $1.044 \mathrm{e}-13$ & $1.492 \mathrm{e}-13$ & $7.994 \mathrm{e}-15$ & $3.109 \mathrm{e}-15$ \\
\hline 0.5 & $3 \cdot 307 \mathrm{e}-08$ & $4.490 \mathrm{e}-08$ & $1.092 \mathrm{e}-13$ & $1.461 \mathrm{e}-13$ & $6.217 \mathrm{e}-15$ & $1.377 \mathrm{e}-14$ \\
\hline 0.6 & $3.425 \mathrm{e}-08$ & $4 \cdot 371 \mathrm{e}-08$ & $1.119 \mathrm{e}-13$ & $1.439 \mathrm{e}-13$ & $7.994 \mathrm{e}-15$ & $2.220 \mathrm{e}-15$ \\
\hline 0.7 & $3.544 \mathrm{e}-08$ & $4.253 \mathrm{e}-08$ & $1.164 \mathrm{e}-13$ & $1.399 \mathrm{e}-13$ & $3.997 \mathrm{e}-15$ & $7.994 \mathrm{e}-15$ \\
\hline 0.8 & $3.662 \mathrm{e}-08$ & $4.135 \mathrm{e}-08$ & $1.226 \mathrm{e}-13$ & $1.332 \mathrm{e}-13$ & $4.885 \mathrm{e}-15$ & $1.288 \mathrm{e}-14$ \\
\hline 0.9 & $3.780 \mathrm{e}-08$ & $4.017 \mathrm{e}-08$ & $1.243 \mathrm{e}-13$ & $1.328 \mathrm{e}-13$ & $9.770 \mathrm{e}-15$ & $1.288 \mathrm{e}-14$ \\
\hline 1.0 & $3.898 \mathrm{e}-08$ & $3.898 \mathrm{e}-08$ & $1.252 \mathrm{e}-13$ & $1.252 \mathrm{e}-13$ & $8.438 \mathrm{e}-15$ & $8.438 \mathrm{e}-15$ \\
\hline
\end{tabular}


As can be seen from both Table 1 and Table 2, the errors become smaller as the stepsize $\mathrm{h}$ becomes smaller. From these results, it can be concluded that ash approaches zero, the approximate solution approaches the exact solutions for both Test Problem 1 and Test Problem 2. It is shown that RK6 approximates FDEs as in Test Problem 1 and Test Problem 2 accurately and the approximate solutions tend to approach exact solutions as the stepsizes becoming smaller.

As for the comparison with existing methods of lower order, the accuracy for RK6 is compared against Euler method and $\mathrm{RK}_{5}$. Numerical results of higher order method produce more accurate results. Table 3 and Table 4describe absolute errors for Euler method, RK5 and RK6 when $h=0.01$ and $r=0.1,0.2, \ldots, 1.0$. For illustrative purposes, only the stepsize $h=0.01$ is shown in this paper. The results for other values of stepsizes also prove to be of the same outcome, it terms of achieving better accuracy as compared with Euler method and RK5.Graphical representation at $t=1$ of the exact solution and approximate solutions using Euler method, RK5 and RK6 for Test Problem 1 and Test Problem 2 are shown in Figure 1 and Figure 2 respectively.

Table 3: Absolute errors of Test Problem 1 for Euler, RK5 and RK6 methods with stepsize $h=0.01$

\begin{tabular}{|c|c|c|c|c|c|c|}
\hline \multirow{2}{*}{$r$} & \multicolumn{7}{|c|}{ Absolute Error } \\
\cline { 2 - 8 } & \multicolumn{2}{|c|}{ Euler } & \multicolumn{2}{c|}{ RK5 } & \multicolumn{2}{c|}{ RK6 } \\
\cline { 2 - 8 } & $\left|Y_{1}-y_{1}\right|$ & $\left|Y_{2}-y_{2}\right|$ & $\left|Y_{1}-y_{1}\right|$ & $\left|Y_{2}-y_{2}\right|$ & $\left|Y_{1}-y_{1}\right| Y_{2}-y_{2} \mid$ \\
\hline 0.1 & $9.911 \mathrm{e}-01$ & $1.904 \mathrm{e}+00$ & $2.926 \mathrm{e}-11$ & $4.200 \mathrm{e}-11$ & $1.632 \mathrm{e}-12$ & $2.347 \mathrm{e}-12$ \\
\hline 0.2 & $9.424 \mathrm{e}-01$ & $1.754 \mathrm{e}+00$ & $3.020 \mathrm{e}-11$ & $4.153 \mathrm{e}-11$ & $1.686 \mathrm{e}-12$ & $2.321 \mathrm{e}-12$ \\
\hline 0.3 & $8.816 \mathrm{e}-01$ & $1.592 \mathrm{e}+00$ & $3.115 \mathrm{e}-11$ & $4.106 \mathrm{e}-11$ & $1.739 \mathrm{e}-12$ & $2.291 \mathrm{e}-12$ \\
\hline 0.4 & $8.073 \mathrm{e}-01$ & $1.416 \mathrm{e}+00$ & $3.209 \mathrm{e}-11$ & $4.058 \mathrm{e}-11$ & $1.792 \mathrm{e}-12$ & $2.266 \mathrm{e}-12$ \\
\hline 0.5 & $7.180 \mathrm{e}-01$ & $1.225 \mathrm{e}+00$ & $3.303 \mathrm{e}-11$ & $4.011 \mathrm{e}-11$ & $1.846 \mathrm{e}-12$ & $2.242 \mathrm{e}-12$ \\
\hline 0.6 & $6.122 \mathrm{e}-01$ & $1.018 \mathrm{e}+00$ & $3.398 \mathrm{e}-11$ & $3.964 \mathrm{e}-11$ & $1.898 \mathrm{e}-12$ & $2.215 \mathrm{e}-12$ \\
\hline 0.8 & $4.882 \mathrm{e}-01$ & $7.925 \mathrm{e}-01$ & $3.492 \mathrm{e}-11$ & $3.917 \mathrm{e}-11$ & $1.950 \mathrm{e}-12$ & $2.186 \mathrm{e}-12$ \\
\hline 0.9 & $3.440 \mathrm{e}-01$ & $5.469 \mathrm{e}-01$ & $3.587 \mathrm{e}-11$ & $3.870 \mathrm{e}-11$ & $2.000 \mathrm{e}-12$ & $2.164 \mathrm{e}-12$ \\
\hline 1.0 & $1.776 \mathrm{e}-01$ & $2.790 \mathrm{e}-01$ & $3.681 \mathrm{e}-11$ & $3.822 \mathrm{e}-11$ & $2.057 \mathrm{e}-12$ & $2.134 \mathrm{e}-12$ \\
\hline
\end{tabular}

Table 4: Absolute errors of Test Problem 2 for Euler, RK5 and RK6 methods with stepsize $h=0.01$

\begin{tabular}{|c|c|c|c|c|c|c|}
\hline & \multicolumn{5}{|c|}{ Absolute Error } \\
\cline { 2 - 8 } & \multicolumn{2}{|c|}{ Euler } & \multicolumn{2}{c|}{ RK5 } \\
\hline & $\left|Y_{1}-y_{1}\right|$ & $\left|Y_{2}-y_{2}\right|$ & $\left|Y_{1}-y_{1}\right|$ & $\left|Y_{2}-y_{2}\right|$ & $\left|Y_{1}-y_{1}\right|$ & $\left|Y_{2}-y_{2}\right|$ \\
\hline 0.1 & $3.063 \mathrm{e}-01$ & $1.780 \mathrm{e}+00$ & $3.931 \mathrm{e}-11$ & $6.882 \mathrm{e}-11$ & $9.370 \mathrm{e}-14$ & $1.625 \mathrm{e}-13$ \\
\hline 0.2 & $3.632 \mathrm{e}-01$ & $1.673 \mathrm{e}+00$ & $4.095 \mathrm{e}-11$ & $6.718 \mathrm{e}-11$ & $9.548 \mathrm{e}-14$ & $1.603 \mathrm{e}-13$ \\
\hline 0.3 & $4.025 \mathrm{e}-01$ & $1.549 \mathrm{e}+00$ & $4.259 \mathrm{e}-11$ & $6.554 \mathrm{e}-11$ & $1.008 \mathrm{e}-13$ & $1.537 \mathrm{e}-13$ \\
\hline 0.4 & $4.229 \mathrm{e}-01$ & $1.406 \mathrm{e}+00$ & $4.423 \mathrm{e}-11$ & $6.390 \mathrm{e}-11$ & $1.044 \mathrm{e}-13$ & $1.492 \mathrm{e}-13$ \\
\hline 0.5 & $4.226 \mathrm{e}-01$ & $1.242 \mathrm{e}+00$ & $4.587 \mathrm{e}-11$ & $6.226 \mathrm{e}-11$ & $1.092 \mathrm{e}-13$ & $1.461 \mathrm{e}-13$ \\
\hline 0.6 & $3.989 \mathrm{e}-01$ & $1.054 \mathrm{e}+00$ & $4.751 \mathrm{e}-11$ & $6.062 \mathrm{e}-11$ & $1.119 \mathrm{e}-13$ & $1.439 \mathrm{e}-13$ \\
\hline 0.7 & $3.482 \mathrm{e}-01$ & $8.395 \mathrm{e}-01$ & $4.915 \mathrm{e}-11$ & $5.898 \mathrm{e}-11$ & $1.164 \mathrm{e}-13$ & $1.399 \mathrm{e}-13$ \\
\hline 0.8 & $2.660 \mathrm{e}-01$ & $5.936 \mathrm{e}-01$ & $5.079 \mathrm{e}-11$ & $5.734 \mathrm{e}-11$ & $1.226 \mathrm{e}-13$ & $1.332 \mathrm{e}-13$ \\
\hline 0.9 & $1.465 \mathrm{e}-01$ & $3.103 \mathrm{e}-01$ & $5.243 \mathrm{e}-11$ & $5.571 \mathrm{e}-11$ & $1.243 \mathrm{e}-13$ & $1.328 \mathrm{e}-13$ \\
\hline 1.0 & $1.797 \mathrm{e}-02$ & $1.797 \mathrm{e}-02$ & $5.406 \mathrm{e}-11$ & $5.406 \mathrm{e}-11$ & $1.252 \mathrm{e}-13$ & $1.252 \mathrm{e}-13$ \\
\hline
\end{tabular}




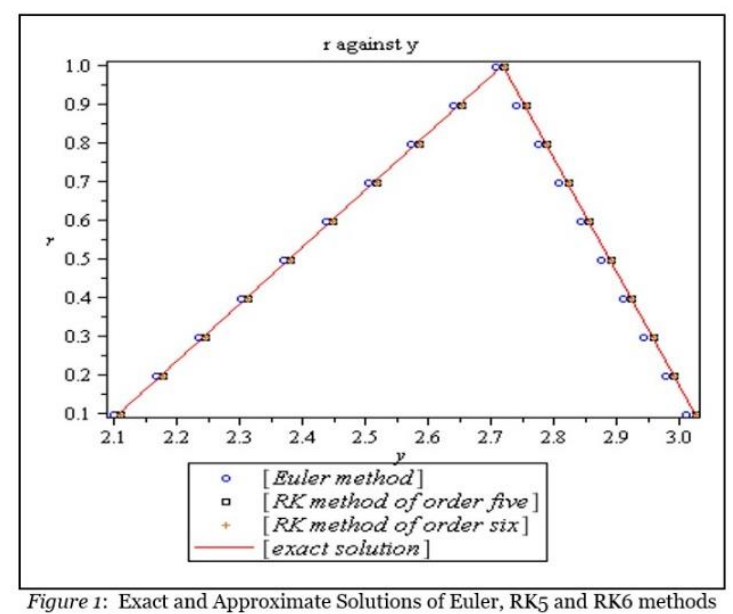

for Test Problem 1 with $h=0.01$

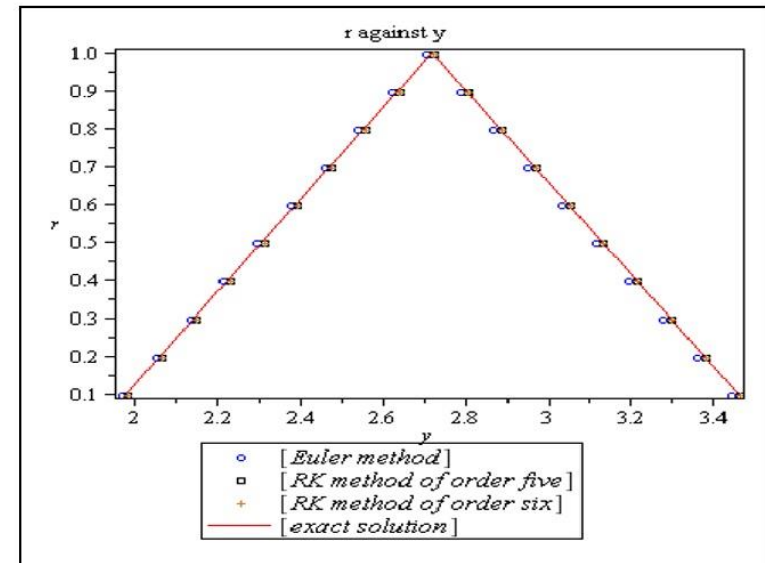

Figure 2: Exact and Approximate Solutions of Euler, RK5 and RK6 metho for Test Problem 2 with $h=0.01$

From Table 3 and Figure 1, it is shown that for $h=0.01$, RK6 solves Test Problem 1 accurately and gives smaller errors as compared with Euler method and RK5. Moreover, RK5 gives smaller errors as compared to Euler method.

From Table 4 and Figure 2, it is shown that for $h=0.01$, RK6 solves Test Problem 2 accurately and gives smaller errors as compared to Euler method and $\mathrm{RK}_{5}$. Moreover, $\mathrm{RK}_{5}$ gives smaller errors as compared to Euler method.

From the numerical results, we can conclude that solving initial value FDEs using RK method of higher order provides more accurate results as compared with methods of lower order. Higher order method is preferable because it is accurate and efficient since computational complexity is minimal due to the higher order method belongs to the same family of one-step methods.

\section{CONCLUSION}

We have presented numerical solutions for initial value FDEs using RK method of order six, RK6. From the numerical results, the method is suitable to solve wide range of FDEs as it gives better accuracy as compared to the existing methods in literature. The method also provides an efficient computation since the stages are all computed in similar manner as other formulae in RK methods. We conclude that RK method of higher order is preferable as it is efficient and achieves the desired accuracy. 


\section{REFERENCES}

Abbasbandy, S \& Viranloo, T. A. (2002), "Numerical solutions of fuzzy differential equations by Taylor method", Computational Methods in Applied Mathematics, vol. 2, no. 2, pp. 113-124.

Ahmad, M. Z., Hasan, M. K. \& De Baets, B. (2013), "Analytical and numerical solutions of fuzzy differential equations", Information Sciences, vol. 236, pp. $156-167$.

Baier, R., Din, Q. \& Donchev, T. (2012), "Higher order Runge-Kutta methods for impulsive differential systems", Applied mathematics and Computation, vol. 218, no. 24, pp. 11790-11798.

Bede, B, Rudas, I. J. \& Benesik, A. L. (2007), "First order linear fuzzy differential equations under generalized differentiability", Information Sciences, vol. 177, no. 7, pp. 1648-1662.

Bede, B. (2013), Mathematics of fuzzy sets and fuzzy logic, Springer-Verlag, Berlin Heidelberg

Butcher, J. C. (1963), “On Runge-Kutta processes of high order", Journal of the Australian Mathematical Society, vol. 4, no. 2, pp. 179-194.

Ismail N., Ibrahim, Z. B.\& Othman, K. I. (2017), “2-point block backward differentiation formulas for solving fuzzy differential equations directly“, Chiangmai Journal Of Science, vol. 44, no. 4, pp. 1781-1791.

Jayakumar, T., Maheskumar, D. \& Kanagarajan, K. (2012), "Numerical solution of fuzzy differential equations by Runge Kutta method of order five”, Applied Mathematical Sciences, vol. 6, no. 6o, pp. 2989-3002.

Jayakumar, T., Raja, C. \& Muthukumar, T. (2014), "Numerical solution of fuzzy differential equations by Adams fifth order predictor-corrector method", Int. Jour. Math. Trends and Technology, vol. 8, pp. 33-50.

Kanagarajan, K. \& Sambath, M. (2010), "Numerical solution of fuzzy differential equations by third order Runge-Kutta method", Int. Journal of Applied Mathematics and Computation, vol. 2, no. 4, pp. 1-8.

Khairul Anuar, K. H., Othman, K. I., Ishak, F., Ibrahim Z. B.\& Majid Z. A.(2011), "Development partitioning intervalwise block method for solving ordinary differential equations“, World Academy of Science, Engineering and Technology, vol. 58, pp. 234-245.

Luther, H. A. (1967), "An explicit sixth-order RungeKutta formula”, Mathematics of Computation, vol. 22, no. 102, pp. 434-436.

Palligkinis, S. C., Papageorgiou, G. \& Famelis, I. T. (2009), "Runge-Kutta methods for fuzzy differential equations", Applied Mathematics and Computation, vol. 209, no. 1, pp. 97-105.

Seikkala, S. (1987), "On the fuzzy initial value problem”, Fuzzy Sets and System, vol. 24, pp. 139-330.

Sulaiman, N. H., Mohamad, D., Mohd Shariff, J., Sayed Ahmad, S. A. \& Abdullah, K. (2018), "Extended FTOPSIS with distance and set theoretic-based similarity measure “,Indonesian Jour. of Electrical Engineering and Computer Science, vol. 9, no. 2, pp. 387-394.

Zadeh, L. (1965), "Fuzzy sets", Information and Control, vol. 3, pp. 338-353.

Zadeh, L. (1975), "The concept of a linguistic variable and its application to approximate reasoning", Information Sciences, vol. 8, pp. 199-249.

Zakaria, N. S. (2016), "Solving fuzzy differential equations using Runge-Kutta method of order six", Master dissertation, Universiti Teknologi MARA, Selangor, Malaysia.

Zimmerman, H. J. (2010), "Fuzzy set theory", Computational Statistics, vol. 2, no. 3, pp. 317-332. 